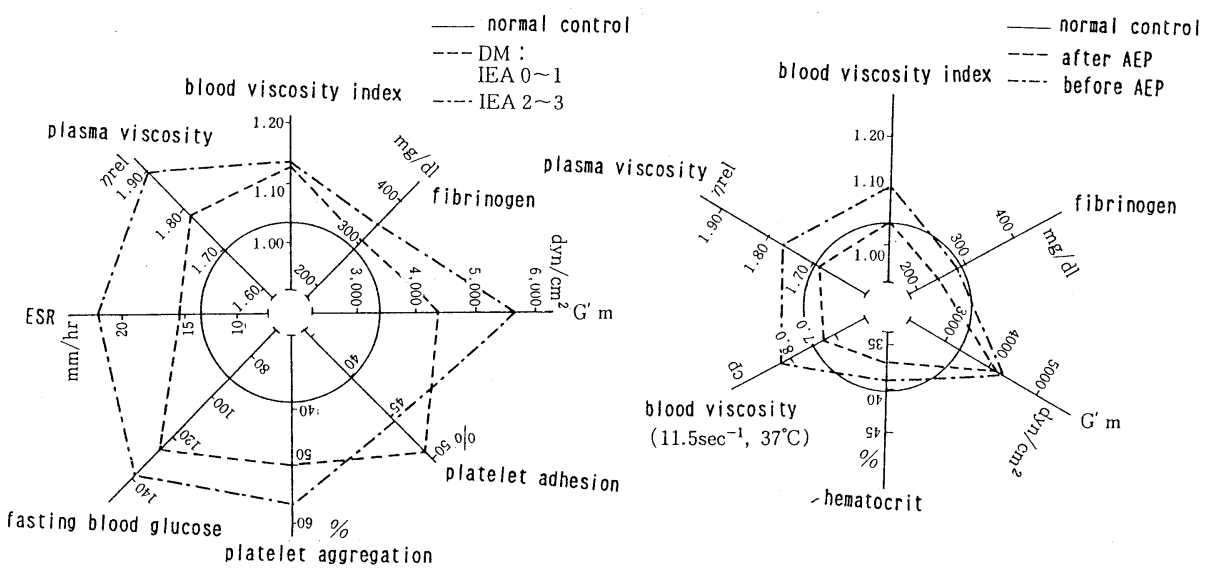

Fig. 2. The left figure shows the hemorheological factors of the two groups into which the diabetics were divided according to the severity of the intravascular erythrocyte aggregation (IEA 0-1: mild aggregation; IEA 2-3: severe aggregation).

The right figure shows the hemorheological factors before and after the application of the artificial endocrine pancreas (AEP).

shift of the distribution, from the D-type to Mtype, and the decrease in the shunt rate. We can conclude from this series of studies that the abnormal hemorheological parameters observed in diabetics have a close relationship with the formation of microthrombi and microangiopathy. Our studies also indicated that those abnormalities can be improved with treatment.

ACKNOWLEDGMENTS: We are most appreciative of the encouragement and scientific advice in the preparation of this manuscript from Dr. Takuo Yokose, Dr.
Takao Shimada and Dr. Masaki Akiyama.

\section{REFERENCES}

1) Isogai $Y$, et al: Dynamic viscoelasticity of blood during clotting in health and disease. Biorheology 10: 411,1973 .

2) Akiyama $M$ : Changes in hemorheological factors in diabetics during application of artificial pancreas. Tokyo Jikeikai Med J 98: 790, 1983 (in Japanese).

3) Shimada T: A study for developing mechanism of diabetic gangrene. Tokyo Jikeikai Med J 99: 747, 1984 (in Japanese).

\title{
(2) Effects of Dynamic and Isometric Exercise on Platelet Function and Blood Coagulability in Cardiac Patients and Normals
}

\section{Kazunobu Yamauchi and Iwao Sotobata}

The First Department of Internal Medicine, Nagoya University School of Medicine, Nagoya

There have been many reports ${ }^{1,2)}$ on the effects of physical exercise upon blood coagulability, and it is said that the enhancement of coagulability and fibrinolysis may be induced by exercise. Recently techniques were developed to assay blood levels of several substances, such as antithrombin
III, protein $\mathrm{C}$, tissue plasminogen activator, and $\alpha_{2}$-plasmin inhibitor, that play roles in controlling hemostasis. The present study aimed to clarify whether exercise-induced alterations in blood coagulability in patients with ischemic heart disease (IHD) or with atrial fibrillation (AF) 
may differ from those in normals. In some patients, the effects of emotional stress on blood coagulability were also investigated.

\section{STUDY POPULATION AND METHODS}

No patients took any drugs that might affect platelet function and blood coagulability for at least 2 weeks before the study. The following studies were performed after informed consent for the procedure was obtained.

Study I Handgrip exercise: IHD patients vs normals

Handgrip exercise at $50 \%$ of maximal voluntary contraction levels were performed for 3 minutes on 9 normal subjects (mean age $47 \mathrm{yrs}$ ) and 10 IHD patients (mean age $55 \mathrm{yrs}$ ). The following items were measured at rest, immediately and at 6 minutes after exercise: platelet counts, ADPinduced platelet aggregation sensitivity ${ }^{3)}, \quad \beta$ thromboglobulin levels, prothrombin time (PT), activated partial thromboplastin time (APTT), activities of factors VIII, IX, XI and XII, and plasma levels of fibrinogen, and antithrombin III.

Study II-1 Treadmill exercise: IHD patients vs normals

Treadmill exercise testing at up to $85 \%$ of the predicted maximal heart rate ${ }^{4)}$ was performed on 10 normal subjects (mean age $54 \mathrm{yrs}$ ) and $10 \mathrm{IHD}$ patients (mean age $56 \mathrm{yrs}$ ). The same items as study I were measured at rest, immediately and at 6 minutes after exercise.

Study II-2 Treadmill exercise: AF patients vs normals

Treadmill exercise testing of the same protocol as study II-1 was performed on 15 normals (mean age $47 \mathrm{yrs}$ ) and 20 patients with lone AF (mean age $49 \mathrm{yrs})$. The measurements were platelet counts, platelet aggregation sensitivities, $\beta$-thromboglobulin, PT, APTT, activities of factors VIII, IX, XI and XII, and levels of protein $\mathrm{C}, \alpha_{2}$-plasmin inhibitor, and $\alpha_{2}$-macroglobulin.

Study II-3 Treadmill exercise: normals

Treadmill exercise testing of the same protocol as study II-1 was performed on 11 normals (mean age $32 \mathrm{yrs}$ ). Factor VIII activities and levels of factor VIII antigen (F VIII C: $\mathrm{Ag})^{5}$, tissue plasminogen activator (TPA), fibrinopeptide A (FPA), fibrinopeptide B $\beta$ 15-42 (FPB $\beta$ 15-42), and Ddimer were measured at rest and immediately after exercise.

Study III Mirror image drawing test: IHD patients

Mirror image drawing test ${ }^{6)}$ was performed on 10 IHD patients (mean age 59 yrs) to induce emotional stress. PT, APTT, levels of fibrinogen and antithrombin III, and activities of factors VIII and IX were measured at rest and immediately after the test.

\section{RESULTS}

Results are summarized in Table 1.

Study I Handgrip exercise: IHD patients vs normals

Platelet counts tended to increase in IHD patients, and $\beta$-thromboglobulin tended to increase in normal subjects after exercise. However, there were no changes in ADP-induced platelet aggregation sensitivity. Fibrinogen levels were higher ( $p$ $<0.01$ ), and antithrombin III levels were lower $(p<0.05)$ at rest in IHD patients than in normal subjects. Both of these substances increased after

Table 1. Effects of treadmill exercise on blood coagulability and platelet function.

\begin{tabular}{|c|c|c|c|c|}
\hline \multirow{2}{*}{ Measurements } & \multicolumn{3}{|c|}{ Treadmill exercise } & \multirow{2}{*}{$\begin{array}{l}\begin{array}{c}\text { Emotional } \\
\text { stress }\end{array} \\
\begin{array}{l}\text { IHD } \\
\text { patients }\end{array} \\
\end{array}$} \\
\hline & $\begin{array}{l}\text { Healthy } \\
\text { controls }\end{array}$ & $\begin{array}{l}\text { IHD } \\
\text { patients }\end{array}$ & $\begin{array}{l}\text { AF } \\
\text { patients }\end{array}$ & \\
\hline Platelet counts & $\nearrow$ & $\nearrow$ & $\uparrow$ & \\
\hline ADP-aggregation & $\rightarrow$ or $\nearrow$ & $\rightarrow$ & $\uparrow$ & \\
\hline$\beta$-thromboglobulin & $\rightarrow$ & $\triangle \rightarrow$ & $\sigma^{7}$ & \\
\hline PT & $\downarrow$ & $\downarrow$ & $\downarrow$ & \\
\hline $\mathrm{APTT}$ & $\downarrow$ & $\downarrow$ & $\downarrow$ & \\
\hline Factor VIII & $\nearrow$ & $\uparrow$ & & $\nearrow$ \\
\hline Factor IX & $\rightarrow$ & $\rightarrow$ & & $\uparrow$ \\
\hline Factor XI & $\rightarrow$ & $\rightarrow$ & & \\
\hline Factor XII & $\nearrow$ & $\uparrow$ & & \\
\hline Fibrinogen & $\nearrow$ & $\hat{0}$ & $\uparrow$ & $\rightarrow$ \\
\hline TPA & $\uparrow$ & & & \\
\hline FPA & $\rightarrow$ & & & \\
\hline FPB $\beta 15-42$ & $\rightarrow$ & & & \\
\hline D.dimer & $\rightarrow$ & & & \\
\hline Antithrombin III & $\uparrow$ & $\hat{\bullet}$ & $\hat{\bullet}$ & $\nearrow$ \\
\hline Protein $\mathrm{C}$ & $\uparrow$ & & $\uparrow$ & \\
\hline$\alpha_{2}$-plasmin inhibitor & $\uparrow$ & & $\uparrow$ & \\
\hline$\alpha_{2}$-macroglobulin & $\nearrow$ & $\uparrow$ & $\uparrow$ & \\
\hline
\end{tabular}

Preexercise comparison between normals and patients is indicated by $\triangle$ : tended to be high; $\circ$ : high; and $\bullet$ : low. The comparison between pre- and post-exercise values is shown by $\rightarrow$ : not significant; $\uparrow$ : tendency to increase; $\uparrow$ : increased; and $\downarrow$ : shortened. 
exercise in both groups. PT and APTT shortened after exercise in IHD patients. Exercise increased factor VIII activities in IHD patients and factor XII activities in normals.

Study II-1 Treadmill exercise: IHD patients vs normals

Platelet counts tended to increase in both groups, and $\beta$-thromboglobulin tended to increase in normals after exercise. Preexercise fibrinogen levels were higher, and antithrombin III levels were lower in IHD patients than in normals $(p<$ 0.05). Both of these substances increased after exercise in both groups. Postexercise activities of factors VIII and XII increased in IHD patients and tended to increase in normals.

Study II-2 Treadmill exercise: AF patients vs normals

There was an increase in platelet sensitivity to ADP-aggregation after exercise in both groups. Preexercise $\beta$-thromboglobulin levels in AF patients were higher than in normals $(\mathrm{p}<0.05)$. Betathromboglobulin increased after exercise in both groups, with the increase being greater in AF patients than in normals (normals: $26 \pm 7 \mathrm{ng} / \mathrm{ml}$ at rest and $35 \pm 14 \mathrm{ng} / \mathrm{ml}$ after exercise; AF patients: $43 \pm 28 \mathrm{ng} / \mathrm{ml}$ at rest and $63 \pm 38 \mathrm{ng} / \mathrm{ml}$ after exercise). PT and APTT shortened, and plasma fibrinogen levels increased after exercise in both groups. Preexercise levels of antithrombin III and protein $\mathrm{C}$ were lower in AF patients than in normals. These two proteins increased after exercise in both groups, and the increase was greater in normals. Plasma $\alpha_{2}$-plasmin inhibitor levels, which were lower in AF patients than in normals at rest increased after exercise in both groups.

Study II-3 Treadmill exercise: normals

Factor VIII activities and levels of factor VIII antigen showed a tendency to increase after exercise. TPA levels were significantly increased from $2.1 \pm 1.2 \mathrm{ng} / \mathrm{ml}$ to $2.6 \pm 1.6 \mathrm{ng} / \mathrm{ml}$ after exercise $(p<0.05)$. However, there were no significant changes noticed in levels of FPA, FPB $\beta 15-42$ and D-dimer with exercise.

Study III Mirror image drawing test: IHD patients

Emotional stress increased the plasma level of epinephrine from $0.04 \mathrm{ng} / \mathrm{ml}$ to $0.10 \mathrm{ng} / \mathrm{ml}$ and that of norepinephrine from $0.35 \mathrm{ng} / \mathrm{ml}$ to 0.50 $\mathrm{ng} / \mathrm{ml}$. Factor VIII activities, and levels of fibrinogen and antithrombin III tended to increase, and factor IX activities increased from $104 \%$ to $110 \%$ by emotional stress.

\section{DISCUSSION}

There were no significant changes observed in platelet counts, sensitivities to ADP aggregation, and $\beta$-thromboglobulin, either in IHD patients or in normals after physical exercise. This finding indicates that platelets are not activated with the treadmill and handgrip exercise performed in the present study. In AF patients, $\beta$-thromboglobulin levels were higher than in normals at rest and increased after exercise. This finding may be related to a higher incidence of systemic embolism in $\mathrm{AF}$ patients ${ }^{7)}$, suggesting the necessity of antiplatelet therapy in patients with high blood levels of platelet specific proteins.

Factor VIII activities increased after handgrip and treadmill exercise in IHD patients and tended to increase in normals, supporting the previous reports ${ }^{8)}$. However, reports on responses of factor VIII antigen to exercise are small in number. In the present study the level of factor VIII antigen tended to increase in normals, suggesting that not only carrying protein but also factor VIII itself increases with exercise.

Fibrinogen levels were higher in IHD patients than in normals before exercise, and a significant increase was noticed after both types of exercise in IHD. Although antithrombin III levels increased after exercise in IHD patients and normals, the pre- and post-exercise levels of antithrombin III were low in the former. In normals treadmill exercise induced an increase in TPA levels without significant changes in levels of FPA, FPB $\beta$ 15-42 and D-dimer. These findings imply that exercise induces activation not only of the coagulationanticoagultion system, but also of the fibrinolysisantifibrinolysis system without loss of hemostatic balance in normals. Although the levels of TPA, FPA, FPB $\beta 15-42$, and D-dimer in IHD or AF were not measured, this may hold true in these patient groups also. However, hemostatic imblance might be induced in cardiac patient groups because of the greater change in platelet function and blood coaguiability accompanied with low 
levels of antithrombin III at rest and after exercise. There was no significant correlation between increases in hematocrit and the level or activity of coagulation factors, suggesting that the increase in these factors may be a response as an acute phase reactant to exercise stress ${ }^{3)}$.

In conclusion, platelet activation by exercise was minimal in normals and IHD patients, but significantly enhanced in AF patients. Blood coagulability and anticoagulability were both accelerated after exercise without loss of hemostatic balance in normals and patient groups. However, there is a possibility that the hemostatic imbalance might be induced by either type of dynamic or isometric exercise, especially in IHD and $\mathrm{AF}$ patients because of the pre- and postexercise hypercoagulability.

ACKNOWLEDGMENT: We would like to thank Prof. Hidehiko Saito for his advice and encouragement and Dr. Hirohiko Furui and Dr. Tadashi Kamiya or excellent technical assistance.

\section{REFERENCES}

1) Bennet B, Ogston D: Physiological variations in co- agulation, fibrinolysis and platelet behaviour. in Ogston D and Bennet B (ed), Haemostasis: Biochemistry, Physiology and Pathology. London, John Wiley \& Sons, 1977, p 354.

2) Lee $G$, et al: Effects of exercise on platelet function and coronary thrombosis. in Mason DT (ed), Advances in Heart Disease, vol 2, New York, Grune Stratton, 1978, p 368.

3) Yamauchi $\mathrm{K}$, et al: Effects of diltiazem hydrochloride on cardiovascular response, platelet aggregation and coagulating activity during exercise testing in systemic hypertension. Am J Cardiol 57: $609,1986$.

4) Sotobata I, et al: Work intensities of different modes of exercise testings in clinical use. Jpn Circul J 43: $161,1979$.

5) Poon MC, et al: Heterogeneity of human circulating anticoagulants against antihemophilic factor (Factor VIII). Blood 46: 409, 1975.

6) Ueno $\mathrm{K}$, et al: The alleviating effects of medazepam on psychologically induced rise in the blood pressure of essential hypertension. Jpn J Clin Pharmacol Ther 8: 17, 1977.

7) Wolf PA, et al: Epidemiologic assessment of chronic atrial fibrillation and risk of stroke: The Framingham Study. Neurology 28: 973, 1978.

8) Iatridis SG, Ferguson JH: Effect of physical exercise on blood clotting and fibrinolysis. J Appl Physiol 8: 337, 1963.

\title{
(3) Hypercoagulative State in Cerebral Thrombosis
}

\author{
Akihiro Igata and Kouichi Shinmyouzu
}

The Third Department of Internal Medicine, Kagoshima University Hospital

Since the final stage of cerebral thrombosis is blood coagulation, the indices of coagulability of the venous blood can contribute to the diagnosis and the evaluation of the effect of treatment or natural course, apart from the arteriosclerosis. From this view, many parameters were checked, such as, blood viscosity, platelet aggregability, $\beta$-thromboglobulin, circulating platelet aggragates (as parameters concerning platelet system), quantitatively and qualitatively changed fibrinogen including, Hageman factor and XIII factor, soluble fibrin monomere complex, $\gamma=\gamma$ dimer and fibrinopeptides (as parameters of coagulative enzyme system), ad von Willebrand factor, and prostacyclin (6 keto-PGF $1 \alpha$ ) (as vascular factors). View- ing all data in patients with cerebral thrombosis, coagulability was confirmed to be very high, and which gradually decreased after onset with or without treatment, i.e. coagulability fluctuates and in the peak of its hyperfunction triggered by some stimuli, thrombosis is induced. Therefore, if the state of hypercoagulability is detected before the onset of stroke, cerebral thrombosis can be obviated through its suppression using suitable drugs. Apart from these parameters, Maruyama found recently that thrombomodulin in the arterioles, plays a role in blood coagulation. Also thrombomodulin was notably found absent in the arterioles of the cerebrum. This, in effect, could offer insights into why cerebral thrombosis 